\title{
Erratum to: Foreword: Special Issue on IPEC 2014
}

\author{
Marek Cygan ${ }^{1}$ • Pinar Heggernes ${ }^{2}$
}

\section{Erratum to: Algorithmica DOI 10.1007/s00453-016-0151-x}

Please note that the correct spelling of the surname of the second author of this Foreword is "Heggernes" (not "Heggerness").

The online version of the original article can be found under doi:10.1007/s00453-016-0151-X.

$\bowtie \quad$ Marek Cygan

cygan@mimuw.edu.pl

1 Institute of Informatics, University of Warsaw, Banacha 2, 02-097 Warsaw, Poland

2 Department of Informatics, University of Bergen, P.O. Box 7803, 5020 Bergen, Norway 